\title{
VISIÓN Y PATRONES DE COMPORTAMIENTO DEL DEPORTE EN ECUADOR: UN ENFOQUE CENTRADO EN LA CULTURA GERENCIAL
}

\section{VISION AND PATTERNS OF BEHAVIOR OF SPORTS IN ECUADOR: A FOCUSED APPROACH TO MANAGEMENT CULTURE}

\section{Summar Alfredo Gómez Barrios, Ph.D. \\ Doctor en Gerencia (Venezuela).}

Docente e investigador de la Universidad Iberoamericana del Ecuador, Ecuador. sgomez@unibe.edu.ec

Pablo del Val Martín, MSc.

Máster universitario en Investigación aplicada a la Educación (España). Docente de la Pontificia Universidad Católica del Ecuador- PUCE, Quito, Ecuador. pdelvalmartin696@puce.edu.ec

\section{ARTíCULO DE INVESTIGACIÓN}

Recibido: 22 de agosto de 2018.

Aceptado: 23 de noviembre de 2018.

\section{RESUMEN}

El objetivo del estudio es identificar la visión y patrones de comportamiento del deporte en ecuador: un enfoque centrado en la cultura gerencial, considerando el deporte de alto rendimiento y las ligas barriales. La investigación implicó la realización de entrevistas en profundidad a personas clave que ejercen cargos gerenciales en estos segmentos deportivos, razón por la cual la metodología se apoyó en un enfoque cualitativo, descriptivo y de campo, materializado en el método de la teoría fundamentada. Los resultados revelaron que existe un quehacer gerencial que en gran medida se ejerce sin las necesarias competencias profesionales, técnicas y humanas, con debilidades en la formación académica y con escasa capacitación en el área gerencial, aspectos que no favorece una cultura gerencial -alineada a una filosofía organizacional- que garantice un desempeño exitoso mediante acciones efectivas para encaminar al deporte ecuatoriano a mejores resultados en el ámbito local, regional e internacional.

Palabras clave: Gerencia; cultura gerencial; organizaciones del deporte; deporte de alto rendimiento; ligas barriales. 


\section{ABSTRACT}

The objective of the study is to identify the vision and behavior patterns of sport in Ecuador: an approach focused on managerial culture, considering high performance sports and neighborhood leagues. The investigation involved conducting in-depth interviews with key people who hold management positions in these sports segments, which is why the methodology was based on a qualitative, descriptive and field approach, materialized in the method of grounded theory. The results revealed that there is a managerial task that is largely exercised without the necessary professional, technical and human skills, with weaknesses in academic training and with little training in the managerial area, aspects that do not favor a managerial culture -aligned to an organizational philosophy- that guarantees a successful performance through effective actions to guide Ecuadorian sports to better results at the local, regional and international levels.

Keywords: Management; managerial culture; sports organizations; High intensity sport; neighborhood leagues.

\section{INTRODUCCIÓN}

Las organizaciones del deporte operan bajo diferentes perspectivas según su finalidad, -como es el caso del deporte de alto rendimiento o el deporte barrial, sin embargo, coinciden en que son estructuras organizativas mediante las cuales se ordena y coordina el trabajo del grupo de personas que cumplen funciones, responsabilidades y tareas según su cargo y jerarquía para lograr los objetivos y metas programadas.

A tal efecto, una organización del deporte orientada bien sea al gran espectáculo competitivo o al deporte para todos sin distingo de sexo, edad, etnia, condición física o mental, no puede lograr aquello que se ha propuesto en términos de cantidad y calidad si las personas dentro de estas instituciones ejercen sus labores de forma individual; más bien deben funcionar bajo un sistema de relaciones alineadas con objetivos claros, con propósitos compartidos y con beneficios comunes, tal como lo afirma Aguilar (2014) enfatizando que una organización del deporte se soporta en “... la actuación conjunta de actores que cumplen funciones, dependiendo de sus cargos y posiciones que ocupan dentro de ella, que adecuadamente articulados proporcionan el logro de los objetivos fijados" (p. 41).

Esta actuación conjunta que indica el precitado autor, debe estar condicionada por una sólida cultura sustentada en aquellos aspectos subjetivos que están presentes en el ser humano como sus actitudes, creencias, valores, motivaciones, hábitos y costumbres, los cuales se 
conectan a través de un sistema de símbolos culturales. Gómez (2017) sostiene que los sistemas simbólicos de la cultura se reflejan a través del comportamiento y de las relaciones de las personas dentro de las organizaciones del deporte, complementando lo siguiente:

Los sistemas simbólicos de la cultura (...) se dan a través de códigos (ajustados a un lenguaje característico y un comportamiento social entre pares, colaboradores y relacionados) como las percepciones, emociones, actitudes, hábitos, creencias, valores, tradiciones, rutinas y otras formas de interacción dentro y entre estos grupos existentes en estas organizaciones, sistemas simbólicos fijados por un conjunto de reglas y normas que deben cumplirse (p. 122)

Adicionalmente, Geertz, (1987) y Schneider, (1980) indican que el comportamiento de los actores sociales y el lenguaje son códigos que se relacionan entre sí y que responden a un sistema de símbolos culturales. Por su parte, Levy-Strauss (1985) y Goodenough (1981) concuerdan que la cultura es la forma como un grupo de personas organizan sus experiencias en torno a una realidad concreta a través de sus percepciones, comprendiendo e interpretando las interacciones cotidianas que orientan el comportamiento de sus miembros. Ello implica el uso de un sistema de símbolos, un lenguaje específico y unas reglas que regulan las relaciones.

Resulta evidente entonces que la cultura es un marco de referencia compartido y aceptado por el grupo de personas de una organización, orientando la manera de pensar y actuar frente a las circunstancias, donde se incluyen aspectos intangibles (actitudes, valores, emociones) y tangibles vinculados a las operaciones cotidianas. Aun así, es una cultura que debe ser atendida y conducida por un quehacer gerencial que además de planificar, estructurar y sistematizar las complejidades que encierra la dinámica organizativa (Kotter, 2007), tenga la capacidad para inspirar a otros; todo ello con el propósito de garantizar la concordancia entre los planes y sus resultados. En sí, una cultura gerencial que ejerza su influencia para movilizar a las personas y por consiguiente a las organizaciones del deporte hacia una visión compartida.

En este orden de ideas, Gómez (2017) revela que las organizaciones del deporte que posean una firme cultura gerencial, están en la capacidad de integrar aspectos como los hábitos, rutinas y modos de conducta aprendidos en la vida organizacional, así como la valoración hacia el trabajo, el acoplamiento de los distintos roles que garantizan la cohesión grupal, la creación de ajustados procesos de comunicación por razones técnicas o por motivos afectivos 
y emocionales, la instauración de un sistema de valores y creencias compartidas, entre otros aspectos.

En torno a los elementos señalados, el objetivo de la investigación es identificar la visión y patrones de comportamiento del deporte en ecuador centrado en la cultura gerencial, considerando dos grandes segmentos: el deporte de alto rendimiento y las ligas barriales. En este sentido, fueron abordados en este estudio actores clave con responsabilidades gerenciales en el deporte ecuatoriano vinculados con estos segmentos, con la finalidad de que pudieran dar cuenta de lo que acontece en la realidad empírica. En definitiva, cuál era su visión con respecto al fenómeno objeto de tratamiento y cómo son los patrones de comportamiento a partir de tres categorías previamente definidas: (a) Perfil Gerencial; (b) Características de la Cultura Organizacional; y (c) Concepción de la Cultura Gerencial.

\section{MATERIALES Y MÉTODOS}

A los efectos de identificar visión y patrones de comportamiento del deporte en Ecuador: un enfoque centrado en la cultura gerencial, la investigación se apoyó en un conjunto de métodos, técnicas y procedimientos durante el proceso que se sintetizan en la figura 1.

Figura 1. Síntesis del encuentro con la realidad.

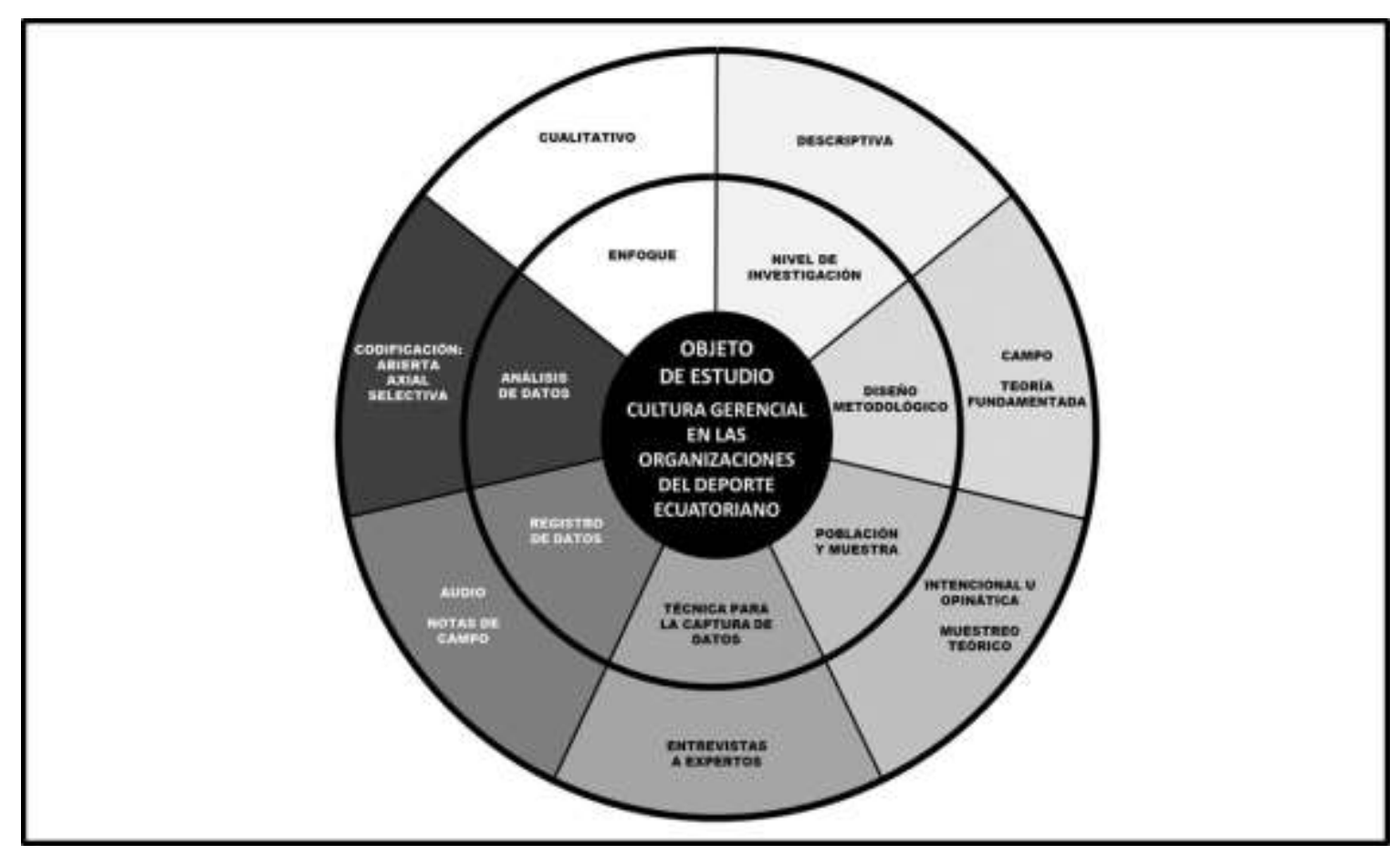

Fuente: Elaboración propia. 
Dado que la realidad es un sistema en permanente interacción y cambio, en este estudio se dispuso asumir el enfoque cualitativo, que a su vez condicionó el tipo de investigación en cuanto a nivel y diseño. El enfoque cualitativo tuvo la intención de analizar e interpretar la realidad desde la perspectiva de los actores sociales, a fin de comprender lo que sucede en el fenómeno objeto de estudio. En este contexto, Rusque (2007) afirma que intentar caracterizar el enfoque cualitativo requiere de sistematización, cuyo interés central es el significado que le otorgan los propios actores a su vida social. Para ello, los principales aspectos que delimitan esta modalidad de investigación están: (a) el mundo social, el sujeto, la subjetividad y la cultura; (b) la actividad que realiza el actor; (c) los procesos en que interactúa; (d) los significados de la acción; (e) las definiciones subjetivas de la acción; y (f) el lenguaje y la comunicación.

En efecto, este enfoque permitió construir el conocimiento mediante las interpretaciones que surgieron del interior de la consciencia subjetiva de los actores sociales. Bajo esta premisa, fue necesario adentrarse en el ambiente donde los sujetos consultados interactúan, con el objeto de recabar datos a partir de sus expectativas, vivencias y experiencias que suscriben sus comportamientos; datos que fueron registrados y utilizados como plataforma para realizar interpretaciones de la concepción que tienen acerca del fenómeno estudiado.

Al mismo tiempo, el estudio se ubicó en una investigación descriptiva mediante la cual se identificaron y analizaron en su justa dimensión aquellos aspectos atinentes al quehacer gerencial que determina la cultura prevaleciente en las organizaciones del deporte ecuatoriano; tipo de investigación que permitió recabar datos de interés para ser conceptualizados, categorizados e interpretados, congruente con las prescripciones de Cerda (1991) quien indica que la investigación descriptiva desde una perspectiva cualitativa, interpreta el comportamiento humano a partir del significado que le otorgan las personas.

Asimismo, el diseño estuvo focalizado en una investigación de campo para tener una relación directa con los actores sociales en su ambiente natural (Arias, 2016), a los efectos de lograr una ajustada interpretación de aquello que es vivido, sentido y percibido por ellos; en sí, su manera de ver y comprender su realidad, cuya interpretación cualitativa de los datos se trianguló con un análisis cuantitativo para fortalecer el estudio (Martínez, 2009).

Para tales propósitos, la investigación se sustentó en la teoría fundamentada de Corbin y Strauss (2002), la cual permitió sistematizar los datos que se iban obteniendo de la realidad. Este método se soportó en cuatro grandes factores: (1) El muestreo teórico; (2) El método comparativo constante; (3) La codificación abierta; y (4) La codificación axial. En la figura 2 se puede apreciar el proceso de análisis sustentado en la teoría fundamentada. 
Figura 2. Proceso de análisis sustentado en la teoría fundamentada.

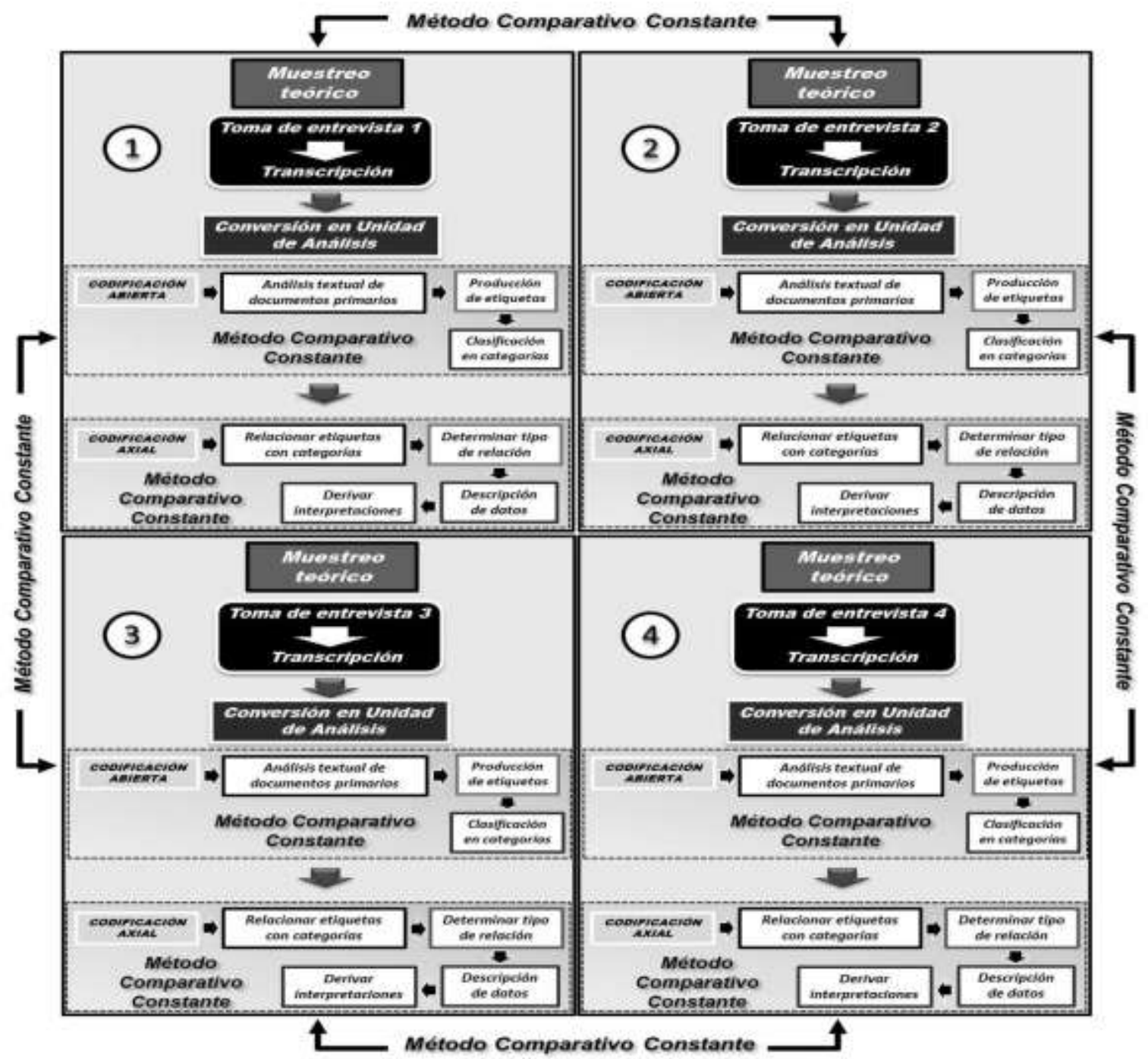

Fuente: Corbin y Strauss (2002)

En torno a lo anterior, la investigación se inició con el muestreo teórico, que consistió en seleccionar de manera intencional a un experto para ser entrevistado en profundidad, cuyo criterio fue que tuviese amplios conocimientos y experiencia en la gerencia del deporte, además de la vivencia en escenarios de competencia como atleta y/o entrenador, y ser profesional universitario.

Una vez realizada y transcrita la entrevista, se configuró como una unidad hermenéutica para ser analizada primeramente en codificación abierta a objeto de fragmentar los datos, identificar conceptos, etiquetarlos y clasificarlos en categorías, y luego en codificación axial para relacionar los conceptos emergentes con sus respectivas categorías a fin de determinar el tipo de relación. Culminado el análisis de la entrevista, se procedió a realizar nuevamente el muestreo teórico con otro entrevistado bajo los mismos criterios de selección y el mismo 
procedimiento en codificación abierta y axial; muestreo teórico que continuó de manera reiterada con otros entrevistados y finalizó cuando hubo saturación teórica, vale decir, cuando no emergieron nuevos datos para ser procesados. Finalmente, los datos se integraron para realizar interpretaciones de los hallazgos encontrados en la realidad empírica.

Cabe indicar que, durante todo el proceso de análisis en ambas modalidades de codificación, se implementó el método comparativo constante dentro de una misma entrevista y entre ellas, de manera de depurar datos que garantizaran el adecuado ordenamiento conceptual en cuanto a conceptualización, categorización y relaciones entre sí. Así pues, la investigación implicó un total de cuatro actores entrevistados con el necesario potencial y relevancia como se muestra en la tabla 1.

Tabla 1. Actores entrevistados en calidad de expertos con base en el conocimiento, la experiencia y la vivencia en el deporte.

\begin{tabular}{|c|c|c|}
\hline ENTREVISTADO & CARGO & RESUMEN DE SU EXPERIENCIA \\
\hline Jorge Granja Cobos & $\begin{array}{l}\text { Vicepresidente de la Federación } \\
\text { Ecuatoriana de Voleibol }\end{array}$ & $\begin{array}{l}\text { Ex atleta y entrenador de selección nacional. Licenciado } \\
\text { en Cultura Física del Instituto Lenin de Moscú. Docente } \\
\text { en la Universidad de San Francisco de Quito. Vinculado } \\
\text { al deporte por } 40 \text { años. }\end{array}$ \\
\hline José Antonio Guevara & $\begin{array}{l}\text { Vicepresidente de la Concentración } \\
\text { Deportiva de Pichincha. } \\
\text { Vicepresidente de la Federación Deportiva } \\
\text { Nacional del Ecuador }\end{array}$ & $\begin{array}{l}\text { Ex atleta. Licenciado en Administración Pública. } \\
\text { Docente de la Universidad Central de Ecuador. Ha sido } \\
\text { miembro de distintas organizaciones del deporte, entre } \\
\text { ellas Vicepresidente del Comité Olímpico Ecuatoriano. } \\
\text { Más de } 50 \text { años relacionado con el deporte. }\end{array}$ \\
\hline Hugo León & Presidente de la Liga Barrial de Quito & $\begin{array}{l}\text { Más de } 60 \text { años en el deporte. Ex atleta de alto } \\
\text { rendimiento y Profesor Normalista. Dirigente en distintas } \\
\text { organizaciones del deporte. }\end{array}$ \\
\hline Pedro Fernández & $\begin{array}{l}\text { Secretario de Educación, Deporte y } \\
\text { Cultura del Distrito Metropolitano de Quito }\end{array}$ & $\begin{array}{l}\text { Abogado con un master en negocios y administración } \\
\text { de fútbol en el Johan Cruyff Institute. Experiencia en } \\
\text { marketing deportivo. Más de } 30 \text { años en el deporte y } \\
\text { actualmente sigue activo como atleta. }\end{array}$ \\
\hline
\end{tabular}

Fuente: Elaboración propia. 


\section{ANÁLISIS DE LOS RESULTADOS}

\subsection{Análisis en codificación abierta y codificación axial}

El proceso de análisis en codificación abierta requirió examinar e interpretar los datos de forma rigurosa y detallada. Debido ello, desde el inicio del análisis textual de los documentos primarios convertidos en unidades hermenéuticas, fueron emergiendo etiquetas conceptuales producto de la fragmentación de estos datos; etiquetas que se clasificaron en tres categorías previamente seleccionadas en función de sus propiedades o atributos, como son:

- Perfil Gerencial: Rasgos asociados al ejercicio gerencial que aseguran comportamientos deseables para un desempeño exitoso.

- Características de la Cultura Organizacional: Atributos de un sistema determinado por el conjunto de relaciones interpersonales existentes en la organización en conexión con la filosofía organizacional.

- Concepción de la Cultura Gerencial: Postura que se tiene respecto a la manera de ver y concebir el quehacer gerencial desde la perspectiva de una cultura orientada a generar cambios personales, grupales y organizacionales.

Esta fragmentación de datos facilitó el ordenamiento conceptual en codificación abierta, que al consolidarlos se generó un total de 147 etiquetas conceptuales agrupadas en las tres categorías aludidas (Tabla 2)

Tabla 2. Ordenamiento conceptual en codificación abierta.

\begin{tabular}{cccc}
\hline UNIDADES & \multicolumn{4}{c}{ CATEGORÍAS } \\
\cline { 2 - 4 } DE ANÁLISIS & $\begin{array}{c}\text { Perfil } \\
\text { Gerencial }\end{array}$ & $\begin{array}{c}\text { Características de la } \\
\text { Cultura Organizacional }\end{array}$ & $\begin{array}{c}\text { Concepción de la } \\
\text { Cultura Gerencial }\end{array}$ \\
\hline Entrevistado 1 & 13 & 17 & 16 \\
\hline Entrevistado 2 & 6 & 9 & 9 \\
\hline Entrevistado 3 & 7 & 12 & 14 \\
\hline Entrevistado 4 & 5 & 22 & 56 \\
\hline TOTAL & 31 & 60 & $38,10 \%$ \\
\hline$\%$ & $21,09 \%$ & $40,81 \%$ & \\
\hline
\end{tabular}

Fuente: Elaboración propia. 
En este sentido, las cifras indican que el $40,81 \%$ de las etiquetas conceptuales fueron clasificadas en la categoría "Características de la cultura organizacional", muy cercana a la categoría "Concepción de la cultura gerencial" con 38,10\%, datos que se reflejaron de manera parecida en cada una de las unidades de análisis.

Estos datos dan cuenta del interés por prevalecer aquellos aspectos referidos a la filosofía organizacional, vale decir cuál es la razón de ser de las organizaciones, cómo se proyectan de cara al futuro y cómo se guía el comportamiento de las personas, en sí el interés en la misión, la visión y los valores organizativos, además de las creencias, las rutinas, los hábitos, entre otros; filosofía organizacional que de igual modo necesita como condición para su cumplimiento de un quehacer gerencial que combine la autoridad y el poder formal que se otorga para planificar, estructurar y sistematizar las complejidades que envuelven la dinámica de una organización con la capacidad para influenciar, persuadir e inspirar a otros. Conforme a esto, una cultura organizacional y gerencial que contribuya a reorientar comportamientos conducentes al logro de los objetivos estratégicos y metas en las organizaciones del deporte ecuatoriano.

Por otra parte, las 147 etiquetas conceptuales que emergieron en codificación abierta fueron analizadas en codificación axial a los efectos de relacionarlas con sus respectivas categorías y determinar el tipo de relación con base en las siguientes prescripciones:

- Es una Propiedad: Rasgos que identifican y se diferencian del resto de los tipos de relación, caracterizando la esencia de un concepto dentro de una categoría.

- Es una Consecuencia: Aquel concepto que se produce como resultado de una acción o hecho derivado de una categoría.

- Es parte: Aun cuando no es un rasgo que lo distingue, es un componente o segmento de un concepto con respecto a la categoría.

- Es contradictoria: Conceptos que discrepan con respecto a la categoría.

Al enlazar estos tipos de relación entre las etiquetas conceptuales y las categorías "Perfil gerencial", "Características de la cultura organizacional" y "Concepción de la cultura gerencial", la tabla 3 muestra cifras que evidencian las tendencias tanto en el total de entrevistados como en cada uno de éstos, dispuestos como unidades de análisis. 
Tabla 3. Relación de etiquetas conceptuales con categorías en codificación axial.

\begin{tabular}{|c|c|c|c|c|c|c|}
\hline & \multirow[b]{2}{*}{ CATEGORÍAS } & \multicolumn{4}{|c|}{ TIPOS DE RELACIÓN DE ETIQUETAS CONCEPTUALES } & \multirow[b]{2}{*}{ TOTAL } \\
\hline & & $\begin{array}{c}\text { Es una } \\
\text { Propiedad }\end{array}$ & $\begin{array}{c}\text { Es una } \\
\text { Consecuencia }\end{array}$ & $\begin{array}{c}\text { Es } \\
\text { Parte }\end{array}$ & $\begin{array}{c}\text { Es } \\
\text { Contradictoria }\end{array}$ & \\
\hline \multirow{5}{*}{ Entrevistado 1} & Perfil Gerencial & 4 & 1 & 5 & 3 & 13 \\
\hline & Características de la Cultura Organizacional & 4 & 1 & 3 & 9 & 17 \\
\hline & Concepción de la Cultura Gerencial & 6 & 2 & 4 & 4 & 16 \\
\hline & TOTAL & 14 & 4 & 12 & 16 & 46 \\
\hline & $\%$ & $30,43 \%$ & $8,70 \%$ & $26,09 \%$ & $34,78 \%$ & $100 \%$ \\
\hline \multirow{5}{*}{ Entrevistado 2} & Perfil Gerencial & 1 & 0 & 1 & 4 & 6 \\
\hline & Características de la Cultura Organizacional & 2 & 0 & 2 & 5 & 9 \\
\hline & Concepción de la Cultura Gerencial & 4 & 0 & 0 & 5 & 9 \\
\hline & TOTAL & 7 & 0 & 3 & 14 & 24 \\
\hline & $\%$ & $29,17 \%$ & $0,00 \%$ & $12,50 \%$ & $58,33 \%$ & $100 \%$ \\
\hline \multirow{5}{*}{ Entrevistado 3} & Perfil Gerencial & 3 & 1 & 1 & 2 & 7 \\
\hline & Características de la Cultura Organizacional & 3 & 1 & 0 & 8 & 12 \\
\hline & Concepción de la Cultura Gerencial & 9 & 1 & 1 & 3 & 14 \\
\hline & TOTAL & 15 & 3 & 2 & 13 & 33 \\
\hline & $\%$ & $45,46 \%$ & $9,09 \%$ & $6,06 \%$ & $39,39 \%$ & $100 \%$ \\
\hline \multirow{5}{*}{ Entrevistado 4} & Perfil Gerencial & 3 & 1 & 1 & 0 & 5 \\
\hline & Características de la Cultura Organizacional & 3 & 6 & 1 & 12 & 22 \\
\hline & Concepción de la Cultura Gerencial & 4 & 2 & 2 & 9 & 17 \\
\hline & TOTAL & 10 & 9 & 4 & 21 & 44 \\
\hline & $\%$ & $22,72 \%$ & $20,46 \%$ & $9,10 \%$ & $47,72 \%$ & $100 \%$ \\
\hline \multirow{5}{*}{$\begin{array}{c}\text { Total } \\
\text { entrevistados }\end{array}$} & Perfil Gerencial & 11 & 3 & 8 & 9 & 31 \\
\hline & Características de la Cultura Organizacional & 12 & 8 & 6 & 34 & 60 \\
\hline & Concepción de la Cultura Gerencial & 23 & 5 & 7 & 21 & 56 \\
\hline & TOTAL & 46 & 16 & 21 & 64 & 147 \\
\hline & $\%$ & $31,29 \%$ & $10,89 \%$ & $14,29 \%$ & $43,53 \%$ & $100 \%$ \\
\hline
\end{tabular}

Fuente: Elaboración propia.

En este tenor, los resultados arrojados en el consolidado de datos de cada una de las entrevistas realizadas a expertos y en el consolidado de datos del total de estas entrevistas, se puede apreciar una inclinación hacia el tipo de relación "Es contradictoria", con un pico de $58,33 \%$ en el entrevistado 2 . De igual forma, en el entrevistado 1 , en el entrevistado 4 y en el total de entrevistados tuvieron $34,78 \%, 47,72 \%$ y $43,53 \%$ respectivamente en este tipo de relación. Solamente en el entrevistado 3 destacó otro tipo de relación: "Es una propiedad" con $45,46 \%$, pero seguido de "Es contradictoria" con 39,39\%.

Estos datos permiten inferir que aun cuando se tiene el deseo de un ejercicio gerencial cuyo perfil debiera estar condicionado por un actor-gerente con las competencias requeridas, matizadas por los conocimientos y las capacidades logradas en las universidades, combinadas a su vez con la experiencia y vivencia en el deporte, la percepción que tienen estos actores de la realidad es más bien un comportamiento gerencial poco cónsono en cuanto al conocimiento y las habilidades que deberían poseer, dado que se adolece de 
formación universitaria para poder conducir las acciones del conjunto de personas adscritas a las organizaciones del deporte en el interés de responder a las necesidades que demanda el sector deportivo en Ecuador. En definitiva, no es suficiente tener la experiencia en campo para dirigir las complejidades que encierra la esfera deportiva, criterio que ha prevalecido en su escogencia.

\subsection{Hallazgos encontrados}

Después de finalizado el proceso de análisis de los datos en codificación abierta y axial de las entrevistas a expertos, donde se logró identificar conceptos, que a su vez fueron etiquetados y clasificados en categorías, y consecutivamente relacionados entre sí para determinar el tipo de relación, se procedió a integrar los datos en cada una de las categorías seleccionadas en este estudio, a los fines de realizar interpretaciones que condujeron a descubrir los hallazgos que surgieron en el proceso investigativo, permitiendo identificar la cultura gerencial en el deporte ecuatoriano, bajo dos vertientes: (1) La visión que tenían los actores; y (2) Los patrones de comportamiento que opera en la realidad. En este tenor, se exponen seguidamente estos hallazgos.

\section{$\underline{\text { Perfil gerencial }}$}

Con respecto a la visión de los actores en esta categoría, se detecta que, para ejercer funciones gerenciales en las organizaciones del deporte, su perfil debe tener como condición una acumulada experiencia en este sector, al igual que conocimientos del deporte a gerenciar, a fin de tener una clara perspectiva de sus necesidades y poder anticiparse a los constantes cambios que se producen en la esfera nacional e internacional. De igual modo, se aspira que el candidato sea profesional universitario y que a su vez posea herramientas gerenciales para maniobrar con propiedad los procesos administrativos; habilidades de liderazgo para influenciar y coordinar esfuerzos con proyección a futuro; y mística deportiva para alcanzar altos niveles competitivos. Adicionalmente, es deseable que un gerente debiera de antemano haber practicado algún deporte; tener relaciones con las federaciones y demás estamentos del deporte; conocer las leyes ecuatorianas, en especial la Ley del Deporte; y poseer ciertos conocimientos sobre marketing deportivo.

En cuanto al comportamiento que opera en la realidad, se revela que existen discrepancias entre la visión de los actores y cómo se ejerce la acción gerencial. En efecto, se sostiene que es práctica común el ejercicio de una gerencia basada en la experiencia que se tiene en el deporte sin la formación requerida para el cargo, además que buena parte de la dirigencia ecuatoriana no posee nivel universitario, acarreando un limitado conocimiento en materia 
gerencial y sin una formación real sobre los procesos administrativos que orienten la conducción idónea de los destinos del deporte, con el agravante que existe en estos cargos personas con un bajo nivel cultural que se traduce en un manejo inadecuado de las relaciones ente pares y colaboradores. Como corolario, se adolece de escuelas de formación en gerencia del deporte. En definitiva, son carencias que en gran medida impiden alcanzar logros importantes y resultados competitivos en escenarios internacionales.

Cabe acotar la afirmación que hace uno de los entrevistados "cuando no existía desarrollo deportivo ¡Quizás era factible un gerente del deporte sin formación!”; afirmación que resulta pertinente, dado los avances en cuanto a funcionamiento, productividad y formas organizativas para gestionar el deporte fundamentado en el conocimiento, razón por la cual es conveniente una gerencia del deporte altamente calificada y centrada en garantizar resultados acordes con los grandes retos que demanda el deporte ecuatoriano.

\section{$\underline{\text { Características de la cultura organizacional }}$}

Como producto de los datos obtenidos de las respuestas de los entrevistados referidos a la visión que tienen sobre esta categoría que estiman deseable para desarrollarse y crecer con éxito, se constata que a las organizaciones del deporte le correspondería involucrar a todos sus miembros en el ánimo de que conozcan en profundidad su filosofía, la cual apunta a la misión, la visión y los valores organizativos, y que a su vez puedan realizar aportes a la dinámica que rodea los procesos, tareas y acciones a seguir alineados con esta filosofía. Estos aspectos en su esencia contribuirían a una mayor identificación institucional, aspectos que resultan muy atinentes con respecto al testimonio de un entrevistado "que se pongan la camiseta, eso falta en muchas organizaciones deportivas". Es evidente entonces que las organizaciones del deporte aspiran una cultura que implique la integración, cohesión, sentido de pertenencia y compromiso ético entre sus miembros y con la organización; una cultura organizacional cuya tendencia esté orientada hacia la práctica de una gestión mucho más profesional, aunando esfuerzos en conjunto y colocando el foco en los resultados que se desean alcanzar.

En relación con el comportamiento que prevalece en las organizaciones del deporte en Ecuador, se confirma que se tiene consolidada una estructura organizativa que parte desde el Comité Olímpico Ecuatoriano y aguas abajo se articula con las federaciones, las ligas, los clubes y los equipos. Asimismo, se valora como parte sustancial de esta estructura, la creación del Ministerio del Deporte, una excelente iniciativa mediante la cual se ha invertido en infraestructura deportiva y se ha instaurado centros de alto rendimiento; aun así, se considera 
que dicha infraestructura no está a la altura de otros países y no se tiene una adecuada preservación y mantenimiento.

Además de lo anterior, este organismo ministerial todavía debe consolidar ciertos procesos como la necesaria atención al deportista élite, no solamente en el aspecto económico que de alguna manera ayuda a paliar algunas necesidades personales para que pueda dedicarse a su preparación, sino también en recibir el primordial acompañamiento de un equipo multidisciplinario vinculado a las ciencias aplicadas al deporte y de entrenadores formados y altamente calificados.

A tal efecto, es conveniente la activación de procesos de capacitación de forma sistemática y permanente en el personal con funciones primarias y de apoyo hacia el atleta; por consiguiente, esta capacitación debe permear todos los niveles jerárquicos de su estructura organizativa, debiéndose complementar con la búsqueda de acuerdos con universidades como por ejemplo la Universidad Tecnológica Equinoccial, institución que tiene el interés en implantar estudios de tercer y cuarto nivel en Administración Deportiva, y la Universidad de las Américas que tiene opciones de estudios en Administración y Marketing Deportivo.

En las mismas circunstancias anteriores, se enfatiza que una importante proporción de los aportes económicos que realiza el Estado ecuatoriano están dirigidos al alto rendimiento, aportes que por demás debieran justificarse con resultados competitivos en el ámbito internacional producto de una planificación con base en varios ciclos olímpicos, como lo hacen las naciones de clases mundial y aquellos países que han tenido progresos importantes en la región. A pesar de ello, los procesos de planificación deportiva ecuatoriana en gran parte no cumplen con esta forma de concebir el futuro, una planificación que abarque dos o tres ciclos olímpicos para estimar hacia dónde se quiere llegar, que incluya un calendario competitivo promovido por el Estado, instando a la gerencia del deporte a que proyecte sus acciones a largo plazo y analizando en conjunto -autoridades deportivas y entes federativos- las expectativas que se desean cumplir. En cualquier caso, es justo reconocer que existen federaciones que están planificando con base en dos ciclos olímpicos y preparándose para las olimpiadas de 2024.

Estas demandas se enfrentan con algunas dificultades, en el hecho que precisamente las autoridades deportivas exigen resultados sin pasar por el tamiz de una evaluación previa, no funciona con planes estratégicos para conducir los objetivos y metas que deban plantearse, y adicionalmente no tienen asesores con pleno conocimiento y experiencia en el deporte, matizados por la presencia de espacios de poder político en distintas instancias, incidiendo de manera importante en las decisiones que se toman; circunstancias que atentan contra el 
necesario respaldo para reconducir acciones que permitan el posicionamiento deportivo a escala internacional. En este mismo sentido, se advierte que no se valora al atleta élite que luego de cumplir años de preparación y participando con éxito en competencias internacionales, al llegar el momento de su retiro no se le presta el apoyo requerido, y en el mejor de los casos se dispensa una atención muy precaria que no ayuda a tener una mejor calidad en el trascurrir de vida.

Por otra parte, desde la perspectiva de los expertos entrevistados se corrobora que las ligas barriales atienden el $40 \%$ de los deportistas ecuatorianos cuyo foco central es mejorar los índices de sedentarismo en la población, incentivando hábitos saludables a través del deporte y la recreación, donde entes gubernamentales han realizado una loable labor con la creación de centros deportivos para atender a este sector, basándose en un modelo de gestión que asegure la preservación de canchas sintéticas con base en la implementación de mecanismos que orienten su adecuado uso, como por ejemplo la fijación de una cuota que favorezca su mantenimiento, eximiendo a aquellos sectores más vulnerables como discapacitados, niños, niñas y mujeres; aspectos que sin duda ayudan a crear conciencia en la gente, todo ello en el interés de que puedan apropiarse de estos espacios. Sin embargo, se aprecia que existen ligas que se encuentran bien dotadas y otras no tanto, producto de políticas deportivas hacia este sector que no están claras, que operan con poco personal y con aportes económicos escasos -en ocasiones con largos periodos sin recibirlos-, motivo por el cual se han visto en la obligación de realizar algunas actividades para cancelar deudas que necesariamente han adquirido.

En este mismo orden de ideas, se revela que el deporte barrial no es una prioridad para el Ministerio del Deporte, más bien se ha centrado exclusivamente en el deporte de alto rendimiento -apreciación que discrepa de otros actores, quienes consideran que se trabaja para el buen vivir en menoscabo del alto rendimiento- y es atendido cuando los políticos están en campaña, a sabiendas que es un nicho que potencialmente genera votos para elegir alcaldes. Se acentúa también que muchos atletas que llegan al alto rendimiento provienen de las ligas barriales, siendo formados con escasos recursos y sus necesidades no son atendidas con efectividad por el Estado "No saben que el atleta sale de aquí ¡De las canchas de tierras!, como Morejón que salió de las canchas de nosotros en pistas de tierras, sin zapatos y esperan que sea campeona mundial para recién reconocerla" manifestaba un entrevistado.

\section{Concepción de la cultura gerencial}

En consideración a la interpretación de los datos obtenidos de las respuestas emitidas por los entrevistados expertos en torno a la visión que tienen acerca de la concepción de la cultura 
gerencial, se deduce que un gerente tiene la obligación de formarse para poder conducir con eficiencia y eficacia las responsabilidades inherentes al cargo. Por supuesto, para ejercer acciones gerenciales, es imperativo que un gerente evalúe permanentemente los procesos básicos de la administración, vale decir, una minuciosa revisión de la planificación, la organización, la dirección y el control de la gestión deportiva. Ello implica saber operar dentro de una estructura organizativa que asegure su solidez, con el liderazgo necesario para influenciar, persuadir y motivar a las personas a través de ajustados procesos de comunicación que tiendan a lograr compromiso en las acciones a seguir y que a su vez deriven en resultados cónsonos con los planes y proyectos en marcha.

Asimismo, un gerente debe ejercer sus funciones, responsabilidades y tareas tanto en la oficina como en el campo, para tener una percepción cercana de la realidad que dé cuenta de lo que allí sucede y pueda estar preparado para enfrentar los retos que se presenten, conociendo de primera mano aquellas inquietudes y necesidades del entorno deportivo como atletas, entrenadores, dirigentes, hinchada y demás grupos de interés por el deporte, sin que ello signifique que no delegue funciones en sus colaboradores para poder contrastar opiniones y considerar distintas alternativas que faciliten la toma de mejores decisiones.

En lo atinente a cómo se concibe la cultura gerencial del deporte sobre la base del comportamiento que opera en estas instituciones, se reafirma que el actor-gerente con formación académica garantizaría en buena parte resultados competitivos. Es este aspecto se reconoce que hay gerentes que poseen esta formación, algunos con nivel de maestría; aun así, no se tiene el suficiente personal con educación universitaria y capacitación en materia gerencial. Debido a ello, la práctica gerencial que hasta ahora ha prevalecido se refleja en la carencia de un pensamiento estratégico para planificar y que oriente las acciones futuras de las personas adscritas a las organizaciones del deporte para cumplir con los objetivos y metas que han sido planificadas.

Es un comportamiento gerencial que se circunscribe a cumplir con un Plan Operativo Anual por exigencias presupuestarias del Ministerio del Deporte, ente administrativo que no funciona con planes estratégicos, y según el criterio de los entrevistados no son los más capacitados, mostrando poco conocimiento sobre el deporte; una alta dirigencia que en sí no tiene una clara visión deportiva. En este sentido, si no hay direccionalidad para controlar la gestión de un Ministerio, menos aún en las demás esferas de la estructura deportiva.

A pesar de todo lo anterior, se constata que algunas federaciones han entendido la importancia de patrocinar sus eventos. A tal efecto, han asumido iniciativas para establecer convenios con empresas que permiten fortalecer la organización de eventos. No obstante, en 
líneas generales se adolece de gerentes con las herramientas necesarias para establecer relaciones que garanticen el patrocinio, y tampoco hay iniciativas para desarrollar el marketing deportivo. Varias de estas herramientas gerenciales que se aluden están vinculadas a competencias blandas basadas en las actitudes, como la perseverancia para lograr los objetivos; la persistencia para superar dificultades; la responsabilidad por el desempeño personal; la disposición para relacionarse, detectar oportunidades, negociar y resolver desacuerdos, entre otras. Ciertamente, se entiende que existe mucho recelo de las empresas para invertir en el deporte porque perciben que no genera rentabilidad; de allí el desafío que tienen los gerentes para construir alianzas o convenios de cooperación con este sector.

Se resalta, asimismo, la conveniencia de observar las mejores prácticas gerenciales en equipos deportivos de clase mundial y de aquellos países de la región como Perú, Venezuela y Colombia, que aventajan a Ecuador en el tema gerencial y que operan con excelentes profesionales con base en su formación académica de tercer y cuarto nivel, además de su comprobada experiencia, obteniendo resultados significativos a escala internacional.

En lo que respecta a las ligas barriales, se asevera que éstas funcionan con un personal gerencial con formación académica como doctores, abogados, administradores, médicos, inclusive ex asambleístas, aunque se confiesa que otros no tienen formación, pero han tenido una ejemplar actuación dentro de esta liga, como lo ilustra un entrevistado "José Francisco Cevallos no tiene título universitario y fue uno de los mejores ministros, y ahora es gobernador. Hay que tener algo dentro para llevar a un equipo como Barcelona a donde está". Es una liga que ha crecido en número de provincias con esfuerzo y autogestión y a pesar de los recortes económicos se sigue trabajando con ahínco.

Es una modalidad de organización la cual entiende que gerenciar el deporte es una tarea ardua y retadora; un esfuerzo que necesariamente tiene que emplear procesos de autogestión para poder funcionar y atender a la inmensa población de atletas que convergen en el deporte barrial. Procesos de autogestión que han permitido adquirir bienes en beneficio de esta colectividad.

\section{CONCLUSIONES}

Los hallazgos que emergieron en este estudio en torno a la visión y patrones de comportamiento del deporte en ecuador centrado en la cultura gerencial, confirman que alto rendimiento y deporte barrial precisan una cultura gerencial sólida que delimite su comportamiento. Una cultura gerencial que se sustente por una parte en el conocimiento logrado tanto en las universidades como en los procesos de capacitación gerencial y 
articulado con la experiencia lograda en años dentro del deporte; y por otra, con aquellos aspectos intangibles y subjetivos que trastocan la dimensión humana. Por consiguiente, un desempeño exitoso dependerá de la combinación de este conjunto de factores que condicionan la cultura gerencial.

Así pues, estos factores adecuadamente alineados bajo un destino compartido -donde exista continuidad administrativa en las altas esferas del deporte para responder a proyectos comunes-, contribuirían al afianzamiento de una sólida plataforma deportiva que permitiese ir edificando las bases para encontrar caminos hacia una ajustada cultura gerencial con un sistema de creencias, actitudes, valores, rutinas y hábitos que ayuden a cambiar el estado actual de cosas en el interés común de transformar definitivamente al deporte ecuatoriano y lo conduzcan hacia el éxito en el contexto local, nacional e internacional.

\section{REFERENCIAS BIBLIOGRÁFICAS}

Aguilar, P. (2014). Gerencia del deporte de alto rendimiento en Venezuela: hacia una implantación efectiva de los procesos administrativos. Saarbrücken: Editorial Publicia.

Arias, F. (2016). El Problema de Investigación: Introducción a la Metodología Científica. Caracas: Editorial Episteme.

Cerda, H. (1991). Los elementos de la investigación. Bogotá: El Búho.

Corbin, J. y Strauss, A. (2002). Bases de la investigación cualitativa: técnicas y procedimientos para desarrollar la teoría fundamentada. Medellín: Universidad de Antioquia.

Geertz, C. (1987). La interpretación de las culturas. México: Editorial Gedisa.

Gómez, S. (2017). Cultura gerencial en las organizaciones del deporte: Valores, creencias, prácticas, herramientas, métodos y procedimientos en organizaciones del deporte. Saarbrücken: Editorial Académica Española.

Goodenough, W H. (1981). Culture, language and society. Reading, Mass: Addison-Wesley.

Kotter, J. (2007). Al frente del cambio. México: Editorial Empresa Activa.

Levy-Strauss, C. (1985). Las estructuras elementales del parentesco. México: Planeta. 
Martínez, M. (2009). Ciencia y arte en la metodología cualitativa. México: Trillas.

Rusque, A. (2007). De la diversidad a la investigación cualitativa. Caracas: Vadel.

Schneider, D. (1980). American Kinship, a Cutural Account. Chicago: The Universisity of Chicago. 